\title{
Transmission Convergence Layer of NG-PON2 in VPIphotonics Tool
}

\author{
Tomas Horvath, Member, IEEE, OSA, Petr Munster, Member, IEEE, OSA, Josef Vojtech, Senior \\ Member, IEEE, OSA, Ondrej Havlis, Member, IEEE, OSA, and Martin Gallo.
}

\begin{abstract}
Nowadays, GPON is the most popular standard for new access networks. However, GPON is able to provide up to 2.5 Gbit/s in both directions in near future customers may require higher bandwidth. The promising solution will be NG-PON2 based networks. This standard is currently popular research field because it is a first standard which completely provides wavelength tuning at ONU. Currently, NG-PON2 standard is interesting for many research groups all around the world because it should provide wavelength tuning at ONU side. The simulation tools such as OptSim, VPIphotonics, and Optiwave support simulation of all types of passive optical networks but only from a physical layer point of view. We successfully implemented transmission convergence layer with all 3 sublayers in cosimulation block in VPIphotonics. Our results prove differences between a fundamental NG-PON2 model and NG-PON2 model with transmission convergence layer. We achieved a minimum of $5 \mathrm{~km}$ extension of ODN network reaches for all attenuation classes (attenuation classes E1 and E2 achieved $10 \mathrm{~km}$ extension of ODN).
\end{abstract}

Index Terms-NG-PON2, Matlab, VPIphotonics, transmission convergence layer, G.989.

\section{INTRODUCTION}

Total Internet traffic has experienced a dramatic growth in the past two decades. According to Cisco VNI (Visual Networking Index): Forecast and Methodology, 2015-2020, the global IP (Internet Protocol) traffic will increase nearly threefold over next couple of years [1]. Until 2020, the annual IP traffic is likely to reach approximately $2.3 \mathrm{ZB}$ essentially resulting in doubling the current broadband speed. Furthermore, the number of devices connected to IP networks is likely to outnumber the global population approximately three times. Therefore, to meet the challenges ahead, the continual industrial development and innovation in this field are essential.

To deal with the higher-bandwidth-consuming applications and services, exponential growth of traffic carried over the network and increasing customer demands for faster and cheaper information, several optical fibre access technologies based on PON (Passive Optical Networks) such as GPON [2] (Gigabit PON), EPON [3] (Ethernet PON) and 10 Gigabit-class PON

Manuscript received May 24, 2017; revised September 29, 2017. Date of publication: October 27, 2017

Tomas Horvath, Petr Munster, Josef Vojtech, and Ondrej Havlis are with CESNET a.l.e., Department of Optical networks, Zikova 4, Prague, and with Department of Telecommunications, Brno University of Technology, Technicka 12, Brno, Czech Republic (E-mail: horvath, munster, havlis, vojtech@cesnet.cz) or (E-mail: horvath, munster@feec.vutbr.cz).

Digital Object Identifier (DOI): 10.24138/jcomss.v13i3.359 technologies XG-PON1 [4] (Next Generation PON), 10GEPON [5] (10 Gigabit Ethernet PON) have been standardized by the ITU-T (International Telecommunication Union) and IEEE (Institute of Electrical and Electronics Engineers) respectively. These PON technologies were designed to operate over a fibre infrastructure based on passive point-to-multipoint power-splitter technology, TDM (Time-Division Multiplexing) and TDMA (Time-Division Multiple Access) mechanisms. However, these standards were designed to use only a single wavelength channel (per-user time slot allocation) to connect the users in downstream (DS) and upstream (US) directions.

Recently, the latest generation PON (NG-PON2) [6], specified in the G.989 series of ITU-T Recommendations, has been defined. In contrast to legacy PON technologies such as GPON [2], XGPON1 [4], etc., NG-PON2 [6] (N-PON Stage 2) is the first PON supporting multiple wavelength channels in DS and US directions offering an accumulated per-PON fibre capacity of $40 \mathrm{Gbit} / \mathrm{s}$ (channel line rate up to $10 \mathrm{Gbit} / \mathrm{s}$ per wavelength) in the DS direction and $10 \mathrm{Gbit} / \mathrm{s}$ in the US direction by taking advantage of WDM (WavelengthDivision multiplexing), channel pairing and colorless tunable transceiver (Tx) technology in the subscriber terminals. NGPON2 can be used by a variety of access, backhaul and fronthaul applications and it is also compatible with legacy powersplit Optical Distribution Networks (ODN) providing reuse of existing technology, installed optical fibre infrastructure and coexistence with deployed legacy PON systems without disruption of customers services especially during upgrade of the network infrastructure.

At present, two major technologies are specified in the NG-PON2 standards: a) hybrid time and wavelength division multiplexing (TWDM) PON [7], [8], [9], and b) wavelength overlay point-to-point wavelength division multiplexing (PtP WDM) PON [6]. The major challenge regarding implementation of both TWDM PON and PtP WDM PON is the efficient, low-cost realization of $\lambda$-tunable transceivers at the optical network units (ONUs) that are essential for realization of modern residential fibre-to-the-home (FTTH) services. Currently, TWDM PON is the primary solution of the NG-PON2.

The main contribution of this paper is own implementation of NG-PON2 TC (Transmission Convergence) layer into VPIphotonics tool. VPIphotonics tool is dedicated for simulations of the physical layer (such as power, attenuation, bit error rate, split ratio etc.). Our implementation proves that TC layer which contains 3 sublayers improve a total system 
reach. Furthermore, our implementation does not require any changes in VPIphotonics tool because we used a co-simulation block with Matlab code.

The rest of this paper is structured as follows. In the next section we get an overview of the related works. Section III presents the the transmission convergence layer of NG-PON2 networks. Section IV describes the simulation scenarios and results discussion. Section V concludes this paper.

\section{RELATED WORKS}

In recent years, many articles about NG-PON2 have been published. Authors [10] deal with optimization of energy reduction with an ILP (Integer Linear Programming). The ILP model is compared with a heuristic algorithm in PON networks based on Ethernet protocol. On the other hand, NG-PON2 does not use message gate for buffer occupation reporting because these networks encapsulate Ethernet frames to own structure. Article [11] focuses on an optimization of activation process for ONUs in NG-PON2 which do not achieve right lambda and transfer data in different channel. We dealt with the activation process in [12] for GPON. Another key area of infrastructure protection for access networks is listed in [13]. The article provides a detailed overview of proposed redundant infrastructure protection topologies, and defines the affected states in the activation process of ONUs. Although redundant network protection schemes were already part of the GPON network, no attention no attention has been paid to this area. A lambda selected ODN idea according to NGPON2 was presented in [14]. Authors compare the usage of lambda selective filters only for a chosen area, however, in other areas a standard power splitter was used. The work [15] presents fast lambda tuning with burst transmission. They use a direct modulation of DBR (Distributed Bragg Reflector) laser with $20 \mathrm{~km}$ reach, low power consumption and stable frequency in operational state in NG-PON2. There are some articles about transmission convergence layer [16], [17] and [18] but current simulation tools are not able to simulate transmission convergence layer because they focus on physical layer parameters (such as power, attenuation, bit error rate, split ratio etc.).

\section{NG-PON2 TRANSMISSION CONVERGENCE LAYER}

TWDM-TC (Transmission Convergence TWDM) layer is composed of three sublayers: a) service adaptation sublayer; b) framing sublayer; and c) physical adaptation sublayer. TWDM-TC layer is present at both the OLT (Optical Line Termination) and the ONU (Optical Network Unit) side. In downstream direction, the interface between TWDM-TC and PMD (Physical Medium Dependent) layers is represented by the bitstream divided into the frames of $125 \mu$ s duration. This process is based on the encapsulation of SDU (Service Data Unit) units that are present in the higher-level layers to the bitstream in the physical layer as depicted in Fig. 1. In the upstream direction, the interface is represented by the series of precisely-synchronized time-bursts.

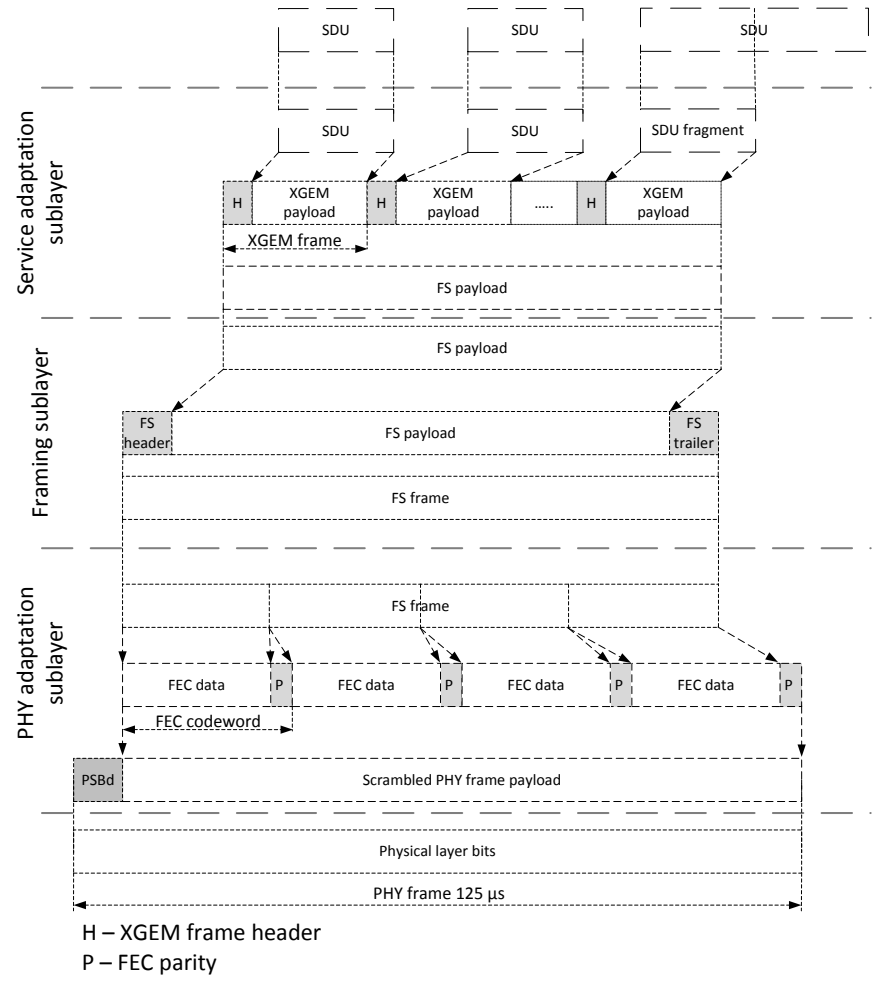

Fig. 1. Downstream SDU mapping [19]

\section{A. Service Adaptation Sublayer}

SAS (Service Adaptation Sublayer) is responsible for encapsulating SDU units from the higher-level layers, multiplexing, and describing the transmitted data. On the transmitter side, this layer accepts SDU units represented by the data from users, ensures the fragmenting process, assigns the XGEM (XG-PON Encapsulation Method) Port-ID to SDU units/fragments, or demands the encapsulation in direction of getting the XGEM frame/s. The XGEM data can be encrypted. Stream of the XGEM frames creates the FS (Framing Sublayer) frame in the downstream direction and the FS burst in the upstream direction. On the receiver side, the SAS accepts the data stored in the FS frames and the FS bursts, respectively. It describes the XGEM frames, filters the frames based on the XGEM Port-ID, decodes the XGEM frames, and demultiplex the SDU units [19].

\section{B. Framing Sublayer}

FS (Framing Sublayer) is responsible for creating and managing the fields necessary for the data-stream management in PON. On transmitter side, this layer accepts the series of XGEM frames composed of FS data, and in combination with the headers/footers addition, it creates the FS frames/bursts in the upstream direction. Logical channels OAM (Operation, Administration and Maintenance), and PLOAM (Physical Layer Operation, Administration and Maintenance) provides information for the management of the data-stream in either direction. In the downstream direction, the FS frame size is flexible. In the upstream direction, it is connected with the PLOAM channel. Furthermore, the data in the FS are 
multiplexed based on the assignment to the Alloc-ID. On the receiver side, this layer accepts FS frames/bursts, analyses the headers, obtains the built-in PLOAM channel, and sends the FS data to the SAS. The information stored in the OAM defines/manages the bandwidth for the upstream direction and controls the timing of the SAS.

\section{Physical Adaptation sublayer}

Physical adaptation sublayer provides function to edit the data-stream modulated by the optical transmitter in direction of improving the detection and sensitivity. It also describes the properties of the signal transmitted through the optical medium, and secures the data by the error-coding techniques. On the transmitter side, this layer accepts the FS frames/bursts from the FS, distributes them into the data-streams for FEC (Forward Error Correction) process. It adds the parity to every block of data, scrambles the FEC blocks, prepares the physical-layer time-synchronization of the bitstream based on PSBd (Physical Synchronization Block appropriate for downstream) or PSBu (PSB appropriate for upstream). On the receiver side, it synchronizes the incoming bitstream, apply the error correction (FEC) and sends the corrected FS frames to the higher-level layers.

\section{Simulation SCHEME}

The simulation scheme used in the simulation model consists of transmitter part OLT, transport part and receiver part ONU. NG-PON2 was deployed in TWDM configuration with 4 lambdas, each one transmitting $10 \mathrm{Gbit} / \mathrm{s}$ in downstream direction from OLT to multiple ONUs. The number of receivers is given by split ratio in 1 or more optical splitters. If more, the partial ratios are multiplied.

Transmitter part on Fig. 2 is created by 4 lasers each representing 1 lambda and combined to WDM. Generation of bitstream and TC layer encapsulation is performed in Matlab and transferred via cosimulation block interface into VPIphotonics scheme where it becomes source of bitstream for modulator. Optical signals are then multiplexed and transferred to optical fibre. Then the common split ratios 1:16, 1:32, 1:64, 1:128, and 1:256 are used. For merely estimated bit error ratio, cosimulation interface has another output with original bit sequence and its brought to the end of scheme where BER (Bit Error Rate) estimation occurs.

ODN part represents passive optical network with passive optical components - fibre and splitters. This simulation network relies on fibre G.657 which is designed for passive optical network with minimum attenuation and high resistance to macrobend.

Receiver side is composed of 2 separate parts. To determine a reliable communication we need high precision of BER and that means as many bits as possible generated in Matlab. Scheme uses also block based on stochastic determined BER for raw BER on Fig. 3. The raw BER is then without any defined encapsulation and error detection coding. This is all done after signal is demultiplexed and usually performed in separate network scheme. The another part on Fig. 4 is created by cosimulation interface which transformed electrical signal

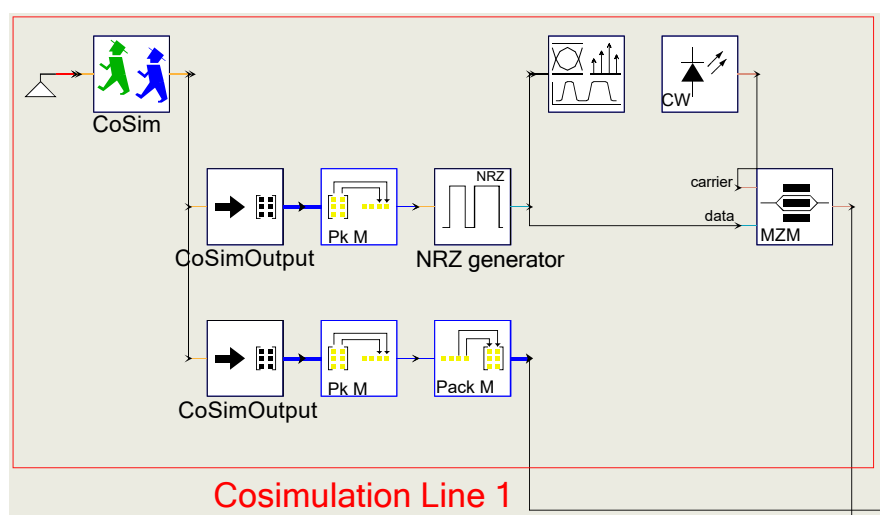

Fig. 2. Transmitter for NG-PON2 in cosimulation mode

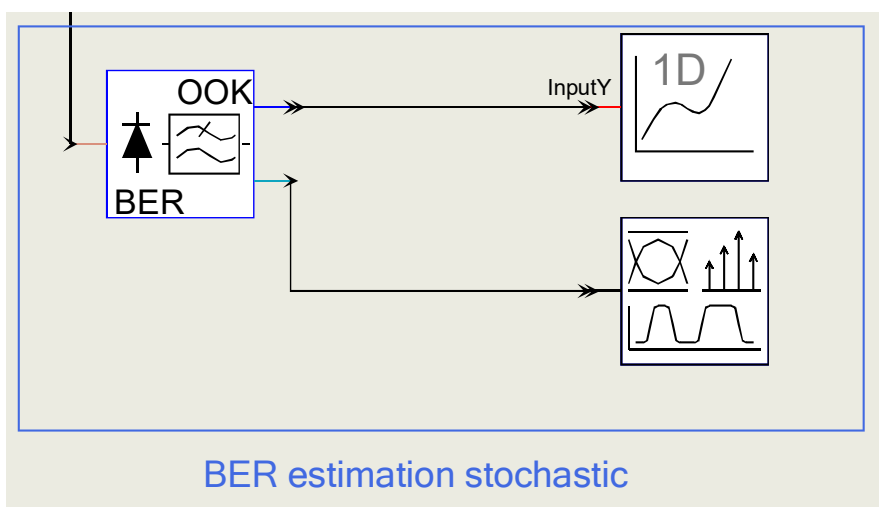

Fig. 3. BER estimation part

from photodiode into the Matlab for further processing and dencapsulation. In both cases, the APD (Avalanche Photodiode) is used [19]. The receiver part represents 1 ONU with 1 lambda only.

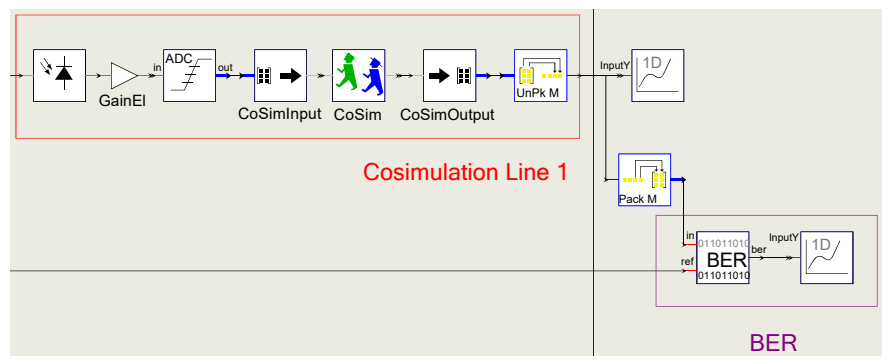

Fig. 4. Cosimulation line on receiver part and BER calculation

\section{A. Implementation of error coding in Matlab}

As mentioned previously, encapsulation and dencapsulation of data stream is performed by Matlab and connected with simulation software VPIphotonics via cosimulation interface. This encapsulation is also represented in Matlab by 3 sublayers on Fig. 5.

Since the size of XGEM frames in third sublayer is different and not the same during encapsulation, the beginning of the program is in the second sublayer known as FS. The number of units is 1083456 bits and header of these FS frames contains 


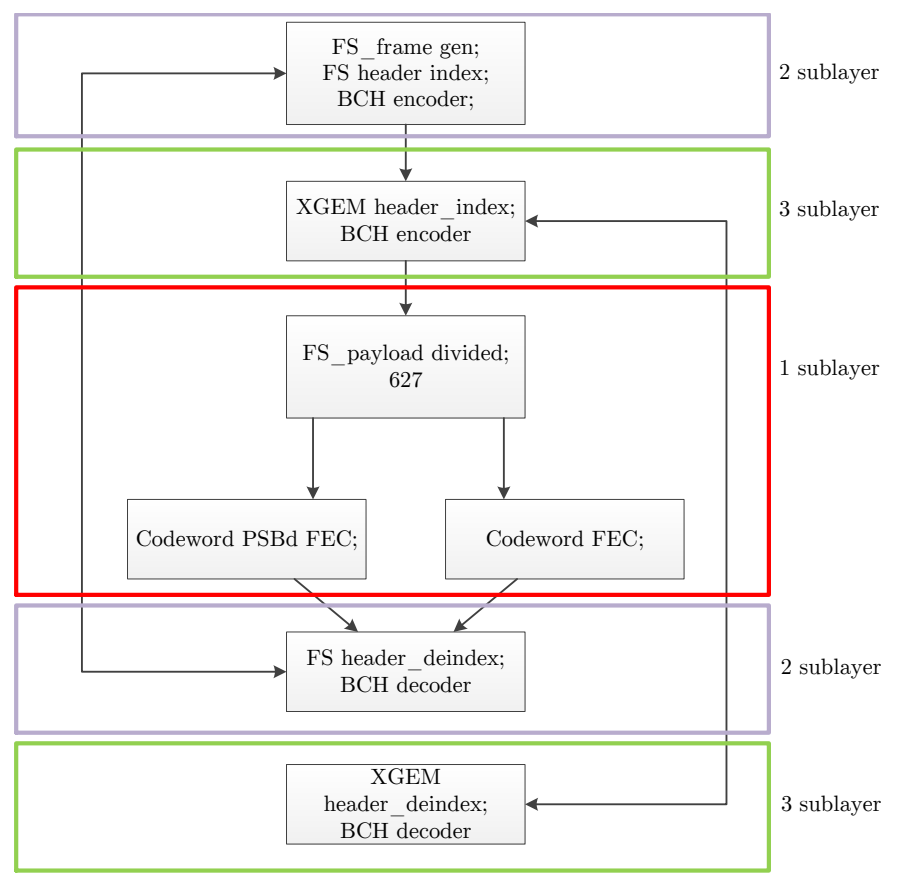

Fig. 5. Sublayer model in Matlab

field and structures and some of them are protected by HEC (Hybrid Error Coding) which is combination of $\mathrm{BCH}$ (Bose, Chaudhuri, and Hocquenghem) codes and parity bit. Payload is then formed by XGEM frames, each frame is indexed and XGEM header is protected by HEC coding. These units of third sublayer known as SAS, contain identifiers necessary for correct delivery of data stream to particular ONU unit and distinguish each service in data flow. Data units of second sublayer are divided into 627 blocks of data and pushed to the first physical sublayer where the main error protection coding occurs and synchronization structures are added. Each block has 1728 bits and after FEC coding becomes codeword with 1984 bits. That means 32 byte parity is added to each block of data. Together with 24 bytes of synchronization PSBd block creates PHY frame as a sum of all 627 codewords and this structure. Situation is illustrated in Fig. 5. At the other end of simulation scheme, there is electrical signal from photodiode converted into bits and dencapsulation with decoding in Matlab is performed. The output bit sequence is compared with the original one from the beginning. Theoretical precision of BER is given by size of FS Frame $-\frac{1}{1083456}$.

\section{B. Simulation parameters}

Standard NG-PON2 provides 4 ODN classes. They vary by sensitivity at photodiode, attenuation of the ODN and values of launched power at R/S point. The parameters for line rate $9.98 \mathrm{Gbit} / \mathrm{s}$ are in Tab. I. Each class can be deployed in various network scenarios with various component requirements.

The launched power is the total power launched to the fibre at the $\mathrm{R} / \mathrm{S}$ point that means 1 lambda is $25 \%$ of that power. In our simulation we used maximum values for all parameters for each class, defined in Tab. II.
TABLE I

ODN CLASSES APRAMETERS IN NG-PON2

\begin{tabular}{|c|c|c|c|c|}
\hline Class & N1 & N2 & E1 & E2 \\
\hline Minimum loss $[\mathrm{dB}]$ & 14 & 16 & 18 & 20 \\
\hline Maximum loss $[\mathrm{dB}]$ & 29 & 31 & 33 & 35 \\
\hline Min power $[\mathrm{dB}]$ & 3 & 5 & 7 & 9 \\
\hline Max power $[\mathrm{dB}]$ & 7 & 9 & 11 & 11 \\
\hline Max sensitivity $[\mathrm{dBm}]$ & -28 & -28 & -28 & -28 \\
\hline Min sensitivity $[\mathrm{dBm}]$ & -7 & -7 & -7 & -9 \\
\hline
\end{tabular}

TABLE II

LAUNCHED POWER OF LIGHT ON 1 LAMBDA USED IN SIMULATIONS

\begin{tabular}{|c|c|c|c|c|}
\hline Class & N1 & N2 & E1 & E2 \\
\hline $1 \lambda$ Power $[\mathrm{dBm}]$ & 1.75 & 2.25 & 2.75 & 2.75 \\
\hline
\end{tabular}

Operating lambdas are shown in Tab. III. For detection of signals, there are avalanched photodiodes used with responsivity (A/W) equals 1 in both scenarios of BER estimation. Optical loss budget of entire scheme is calculated as a sum of particular optical components. Together with min and max sensitivity, they have to match values from Tab. I. Attenuation of multiplexer and demultiplexer is $0.8 \mathrm{~dB}$ per port and modulator has $3.1 \mathrm{~dB}$. Optical fibre G.657.A has attenuation coefficient $0.22 \mathrm{~dB} / \mathrm{km}$ at $1625 \mathrm{~nm}$. ODN classes $\mathrm{N} 1, \mathrm{~N} 2$ and $\mathrm{E} 1$ are used in configuration with this type of fibre. In our simulation model class E2 uses modern ALLwave ${ }^{\circledR}$ fibre with attenuation less than $0.20 \mathrm{~dB} / \mathrm{km}$ for $1625 \mathrm{~nm}$. The rest of parameters are in [20].

TABLE III

SIMULATION LAMBDAS USED IN SIMULATION MODEL

\begin{tabular}{|c|c|c|}
\hline Channel & Central frequency [THz] & Wavelength [nm] \\
\hline 1 & 187.8 & 1596.34 \\
\hline 2 & 187.7 & 1597.19 \\
\hline 3 & 187.6 & 1598.04 \\
\hline 4 & 187.5 & 1598.89 \\
\hline
\end{tabular}

The precision of simulation is highly dependent on setting of TimeWindow parameter. It represents amount of data passing through optical blocks in block mode simulation. In our case this parameter was set to maximum permissible value when simulation was still stable, $2 \cdot 10^{20}$. By running simulation in interactive mode multiple times, the BER precision is higher by number of repetitions. Since transferred bits create PHY frame with error protection redundancy included, the real BER is given by size of user data in FS Frame on the second sublayer. For this purpose, FS frame had to be reduced and the resulting BER is then $2.192 \cdot 10^{-7}$ and each higher value is shown as zero and thanks to cooperation with scheme without error protection and limited BER we can decide if communication in downstream is stable, reliable, and if operates or not.

\section{Simulation results}

Simulations were performed for all ODN classes and various lengths of fibre. We compared ODN with same split ratio with and without our implementation of FEC according to [19] from 
fibre distance point of view by limited BER value (without $\mathrm{FEC}=10^{-12}$ and with $\mathrm{FEC}=10^{-4}$ ). The first split ratio $1: 16$ is not often used in real application, but can provide good grounding about maximum fibre reachable distance, and also test higher values in receiving power on photodiode near to maximum overload sensitivity. The ODN classes N1 and N2 where less powerful and cheaper lasers are used can provide us reliable communication even without any FEC error coding up to 25 and $30 \mathrm{~km}$. With this feature on the fibre, lengths are improved by $60 \%$ total reach is more than $40 \mathrm{~km}$. Class E1 with maximum optical power ensure reliable communication on $40 \mathrm{~km}$ without FEC and $55 \mathrm{~km}$ with error coding on. The least likely usable scenario at first sight over this split ratio and ODN class E2 with special ALLwave ${ }^{\circledR}$ fibre is able to reach $60 \mathrm{~km}$ with FEC error correction coding. This scenario is more suitable for utilization in mobile networks when operator can choose to deploy passive optical network to interconnect particular mobile network elements.

The next ratios 1:32 and 1:64 represent higher probability in deployment as passive optical network in these numbers of receivers each receiving various services. Fibre distance without FEC error detection coding in 1:32 vary from 20 to $30 \mathrm{~km}$. Turning off this feature leads to increase throughput ready for user traffic with same bandwidth $9.98 \mathrm{Gbit} / \mathrm{s}$. As was mentioned before FEC parity takes about $13 \%$ of the bandwidth. This is done automatically by reporting average BER in upstream 2nd sublayer units - FS bursts. Turning on error correction coding causes extension from $30 \mathrm{~km}$ to $40 \mathrm{~km}$ for $30 \mathrm{~km}$ to $45 \mathrm{~km}$, respectively. One of the most common splitting ration is $1: 64$. ODN class $\mathrm{N} 1$ is almost hardly applicable with reliable transmission over $10 \mathrm{~km}$. Network provider needs to deploy network with at least ODN class N2 and higher. Standard NG-PON2 in DD40 configuration should be able to ensure reliable transmission over the fibre length of $40 \mathrm{~km}$. However our simulation scenario shows us it is possible only with fibre which is $5 \mathrm{~km}$ shorter. Avalanche photodiode and inner processing circuits of this simulation network are able to process optical power in acceptable BER with receiving optical power no less than $-25 \mathrm{dBm}$. Standard counts with very courageous $-28 \mathrm{dBm}$ and so there is a part for improvements either in reduction total loss budget or more likely in using more sensitive APD receiver. Our simulation software did not provide that - responsivity (A/W) was set to 1 . Second option is using passive fibre extender based on the Raman amplification [21]. The passive nature of network remains unchanged but other things bounded with this solution must be take into consideration. The last 2 split ratios 1:128 and 1:256 and their BER dependence on fibre distance on Fig. 7 provide high number of potential recipients. However their deployment is eligible with ODN class N2 and higher. The transmitting power in this class provide us reliable communication only at $10 \mathrm{~km}$ fibre length with FEC on. Ratio 1:256 has limited real use on longer distances and can be deploy only with mentioned fibres and power budget at distance $10 \mathrm{~km}$ with FEC on. For now, provider must use more OLT transmitting units in order to satisfy demand of this high number of recipients on longer distances and still using passive optical solution.

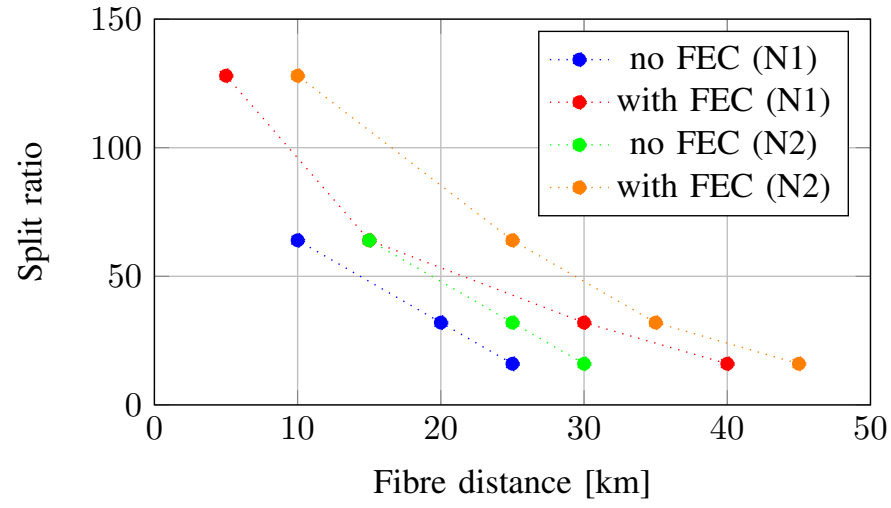

Fig. 6. Reliable transmission at N1 and N2 ODN classes.

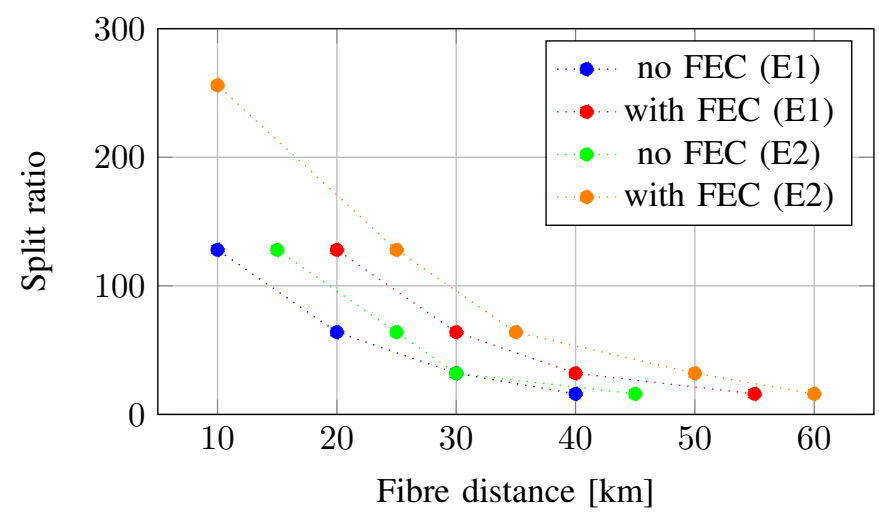

Fig. 7. Reliable transmission at E1 and E2 ODN classes

Finally, we compared two eye diagrams for 1:64 split ratio with and without FEC for $35 \mathrm{~km}$ length of ODN. As can be seen in Fig. 8 the eye diagram with FEC implementation has better parameters. For example, the logical level of 0 below in Fig. 8 and nearly to the $\mathrm{x}$-axis is approximately same for both eye diagrams with small ISI (Inter-Symbol Interference). In other words, line widths are same in comparison with lines for the logical level of 1 in Fig. 8 nearly to the y-axis. There is the main difference in line width. The eye diagram for simulation without FEC has a bigger line width which causes higher ISI. Inter-Interference Interference (ISI) is a form of distortion caused by a flawed sequence of symbols. This is an undesirable effect on BER in the receiver, which can be reduced by a correction code. The lower ISI for the logical level of 1 proves a proper function of our TC layer implementation.

\section{CONCLUSION}

In this paper, we briefly introduced NG-PON2 standard of access networks. We also focused on an implementation of transmission convergence layer for these networks. However, all commercially available optical network simulation tools primarily focus on a physical layer aspect, they also support a co-simulation block with Matlab code and/or TCL (Tool Command Language) language. Our implementation uses a Matlab code and proofs a correct function.

The simulation results show differences in same NG-PON2 topology and parameters with and without a transmission layer implementation. In other words, results without transmission 


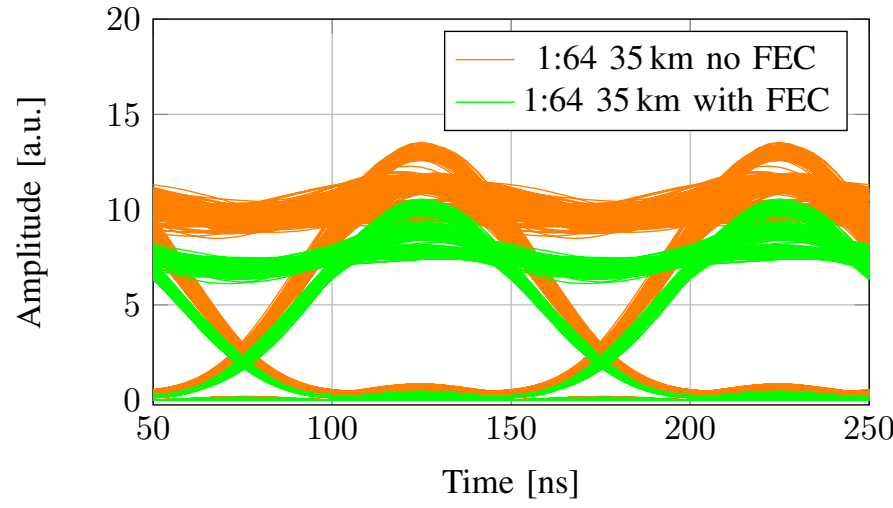

Fig. 8. EYE diagrams for $35 \mathrm{~km}$ length of ODN with and without FEC.

convergence layer implementation are not able to provide satisfactory values because they do not use encapsulation method and a correction mechanism for data transmission. For example, according to simulations without TC layer of NGPON2 is able to transmit data up to $10 \mathrm{~km}$ (N1 attenuation class) for 64 customers but with a TC layer implementation is $15 \mathrm{~km}$ for 64 customers. We achieved a minimum of $5 \mathrm{~km}$ extension of ODN network reaches for all attenuation classes. In general, results are strongly dependent on TC layer implementation.

The future work will continue with modifying our implementation of NG-PON2 TC layer for IEEE PONs which are based on Ethernet frames transmission.

\section{ACKNOWLEDGMENT}

Presented research has been supported by project "Einfrastructure CESNET modernisation", registration no. CZ.02.1.01/0.0/0.0/16 013/0001797 and the National Sustainability Program under grant LO1401. For the research, infrastructure of the SIX Center was used.

\section{REFERENCES}

[1] Cisco: The Zettabyte Era - Trends and Analysis - Cisco, 2016. Available: https://goo.gl/dAO7EY

[2] G.984.1: Gigabit-capable passive optical networks (GPON): General characteristics, International Telecommunication Union, 2008. Available: https://www.itu.int/rec/T-REC-G.984.1/en.

[3] 802.3ah-2004 - IEEE Standard for Information technology Local and metropolitan area networks - Part 3: CSMA/CD Access Method and Physical Layer Specifications Amendment: Media Access Control Parameters, Physical Layers, and Management Parameters for Subscriber Access Networks, Institute of Electrical and Electronics Engineers, 2004. Available: http://www.ieee802.org/21/doctree/2006_Meeting_Docs/200611 meeting docs/802.3ah-2004.pdf.

[4] G.987.1 : 10-Gigabit-capable passive optical networks (XG-PON): General requirements, International Telecommunication Union, 2010. Available: https://www.itu.int/rec/T-REC-G.987.1/en.

[5] Physical Layer Specifications and Management Parameters for $10 \mathrm{~Gb} / \mathrm{s}$ Passive Optical Networks, Institute of Electrical and Electronics Engineers, 2009.

[6] G.989.1 : 40-Gigabit-capable passive optical networks (NG-PON2): General requirements, International Telecommunication Union, 2013. Available: https://www.itu.int/rec/T-REC-G.989.1/en.

[7] Y. Luo, X. Zhou, F. Effenberger, X. Yan, G. Peng, Y. Qian, and Y. Ma, Time- and Wavelength-Division Multiplexed Passive Optical Network (TWDM-PON) for Next-Generation PON Stage 2 (NG-PON2), Journal of Lightwave Technology, vol. 31, no. 4, pp. 587-593, 2013.
[8] D. van Veen, W. Poehlmann, B. Farah, T. Pfeiffer, and P. Vetter, Measurement and Mitigation of Wavelength Drift due to Self-Heating of Tunable Burst-Mode DML for TWDM-PON, in Optical Fiber Communication Conference, 2014, p. W1D.6-.

[9] R. Murano, Optical Component Technology Options for NGPON2 Systems, in Optical Fiber Communication Conference, 2014, p. M3I.1-

[10] R. Wang, P. Bhaumik, H. H. Lee, S. S. Lee, and B. Mukherjee, Energy Management in NG-PON2, in Optical Fiber Communication Conference, 2014, p. Tu3C.4-

[11] L. Bertignono, S. Capriata, V. Ferrero, L. Greborio, R. Mercinelli, M. Valvo, and R. Gaudino, Photon ranging techniques for upstream signalling in TWDM-PON during ONU activation, in 2015 European Conference on Optical Communication (ECOC), 2015, pp. 1-3.

[12] T. Horvath, P. Munster, M. Jurcik, and M. Filka, Novel Algorithm in Activation Process of GPON Networks, Journal of Communications Software and Systems, vol. 11, no. 4, pp. 204-208, Mar. 2016.

[13] Y. Luo, B. Gao, M. Jiang, and F. Effenberger, N: 1 fast protection in NG-PON2, in 2015 Opto-Electronics and Communications Conference (OECC), 2015, p. 1-3.

[14] K. Grobe, WDM-PON with Wavelength-Routed ODN - Pros'n'Cons, in Photonic Networks; 16. ITG Symposium; Proceedings of, 2015, p. 5157.

[15] Y. Matsui, W. Li, H. Roberts, H. Bulthuis, H. Deng, L. Lin, and C. Roxlo, Transceiver for NG-PON2: Wavelength Tunablity for Burst Mode TWDM and Point-to-point WDM, in Optical Fiber Communication Conference, 2016, p. Tu2C.1-

[16] Y. Luo, H. Roberts, K. Grobe, M. Valvo, D. Nesset, K. Asaka, H. Rohde, J. Smith, J. S. Wey, and F. Effenberger, Physical Layer Aspects of NG-PON2 StandardsPart 2: System Design and Technology Feasibility [Invited], Journal of Optical Communications and Networking, vol. 8 , no. 1, p. $43-, 2016$

[17] D. Nesset, NG-PON2 Technology and Standards, Journal of Lightwave Technology, vol. 33, no. 5, p. 1136-1143, 2015-3-1.

[18] D. A. Khotimsky, NG-PON2 Transmission Convergence Layer: A Tutorial, Journal of Lightwave Technology, vol. 34, no. 5, pp. 1424-1432, 2016-3-1.

[19] G.989.3 : 40-Gigabit-capable passive optical networks (NG-PON2): Transmission convergence layer specification, International Telecom munication Union, 2013. Available: https://www.itu.int/rec/T-RECG.989.3/en.

[20] Furukawa: AllWave ${ }^{\circledR}$ Fiber - Zero Water Peak, Furukawa, 2016 Available: https://goo.gl/1QYxRs.

[21] B. Zhu, Entirely passive reach extended GPON using Raman amplification, Optics Express, vol. 18, no. 22, pp. 23428-23434, 2010.

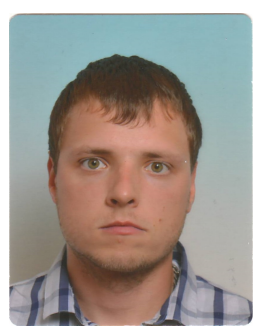

IEEE and OSA.
Tomas Horvath (MSc, Ph.D.) was born in 1989, in Havirov (Czech Republic). He is a young researcher at Brno University of Technology and CESNET a.l.e. $\mathrm{He}$ received his Ph.D. at the Brno University of Technology, Department of Telecommunications in 2017 on the thesis entitled Services Optimization in FTTx Optical Access Networks. His record shows more than 35 peer reviewed proceedings and journal papers. His current research interests include passive optical networks, software defined optical networking, and sensing. He is a student member of SPIE,

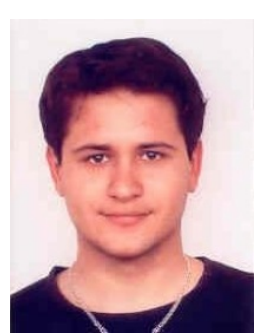

Petr Munster (MSc, Ph.D.) was born in 1984 , in Zlin (Czech Republic). He received his Ph.D at the Brno University of Technology, Department of Telecommunications in 2014 on the thesis entitled Parameters of the FTTx networks. His current research themes focus on fibre-optic sensors, especially distributed fibre-optic sensors, and also on fibre-optic telecommunications. He has abou 50 scientific publications in journals and conferences in last 5 years. 


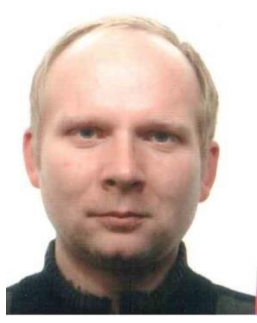

Josef Vojtech is a head of research department of Optical networks at CESNET a.l.e., operator of national research and education e-infrastructure of the Czech Republic. He received his MS and BS degrees in computer science and pedagogy from the Czech Technical University in 2001 and 2003, respectively, and his $\mathrm{PhD}$ degree in field of optical networking from the Czech Technical University in 2008. His record shows more than 65 peer reviewed proceedings and journal papers. He holds 18 patents (including US and EU) and utility models. He participated in international projects: COMPLETE, FI-PPP XIFI, GN4, GN3+, GN3, GN2, Porta Optica Study and SEEFIRE. His current research interests include software defined optical networking and special photonic services as precise time, stable frequency or sensing. He is a member of SPIE, IEEE and OSA. In 2007 he received the Research prize of the Czech minister of education.

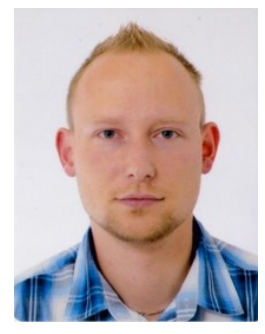

Ondrej Havlis received the Master's degree in the field communications and Informatics science from the Brno University of Technology, in 2012. He joined Optical networks department of CESNET, a.l.e., in 2012. He is active in research, design and verification of Photonic Services in the optical networks and development Photonic Testbed. He was actively involved in the project GN3 and GN3plus. In 2015 Ondrej has started to study at Brno University of Technology at Faculty of Electrical Engineering and Communication $\mathrm{PhD}$ degree with topic of this name Effective Optical Function in Fibre Photonic Networks. Currently Ondrej participates in the project threat detection of infrastructure safety.

Martin Gallo (MSc) Martin Gallo (MSc) was born in Slovakia and received his BS and MSc degrees in Communications and Informatics from Brno University of Technology in 2016. After his work for AT\&T in a field of mobile networking, his current work in Deutsche Telekom includes testing new radio technologies for 5G NR network as well as IoT world and proposing innovations of RAN and core network for all DT group. 